\title{
Erratum to: Updated numerical integration method for stability calculation of Mathieu equation with various time delays
}

\author{
Xiao Jian Zhang • Cai Hua Xiong • Ye Ding • \\ Han Ding
}

Published online: 16 January 2017

(C) Springer Science+Business Media Dordrecht 2017

\section{Erratum to: Nonlinear Dyn \\ DOI 10.1007/s11071-016-3096-3}

The authors regret there is a negligence in Sects. 2.1 and 3 in the original publication.

The solution of Eq. (2) in Sect. 2.1 can be expressed in the integral form when $\mathbf{A}(t)$ is a constant matrix

$$
\begin{aligned}
\mathbf{x}(t)= & \mathrm{e}^{\mathbf{A}(t) \cdot\left(t-t_{1}\right)} \mathbf{x}\left(t_{1}\right) \\
& +\sum_{j=1}^{s} \int_{t_{1}}^{t} \mathrm{e}^{\mathbf{A}(t) \cdot(t-\xi)} \mathbf{B}_{j}(\xi) \cdot \mathbf{x}\left(\xi-\tau_{j}\right) \mathrm{d} \xi,
\end{aligned}
$$

where $\mathbf{x}\left(t_{1}\right)$ denotes the state value at $t=t_{1}$.

At each small subinterval $\left[t_{i-1}, t_{i}\right]$, Eq. (3) is represented as

$$
\begin{aligned}
\mathbf{x}(t)= & \mathrm{e}^{\mathbf{A}(t) \cdot\left(t-t_{i-1}\right)} \mathbf{x}\left(t_{i-1}\right) \\
& +\sum_{j=1}^{s} \int_{t_{i-1}}^{t} \mathrm{e}^{\mathbf{A}}(t) \cdot(t-\xi) \mathbf{B}_{j}(\xi) \\
& \cdot \mathbf{x}\left(\xi-\tau_{j}\right) \mathrm{d} \xi, \quad t \in\left[t_{i-1}, t_{i}\right] .
\end{aligned}
$$

The online version of the original article can be found under doi:10.1007/s11071-016-3096-3.

\section{J. Zhang · C. H. Xiong $(\varangle) \cdot$ H. Ding}

State Key Laboratory of Digital Manufacturing Equipment and Technology, School of Mechanical Science and

Engineering, Huazhong University of Science and

Technology, Wuhan 430074, China

e-mail: chxiong@hust.edu.cn

Y. Ding

State Key Laboratory of Mechanical System and Vibration, School of Mechanical Engineering, Shanghai Jiao Tong University, Shanghai 200240, China
It is assumed that $\mathbf{A}(t)$ is a constant matrix on the small interval $t \in\left[t_{i-1}, t_{i}\right]$. For convenience, $\mathbf{A}(t)$ is replaced with $\mathbf{A}\left(t_{i}\right)$ as an approximation. For the corresponding discretized time points $t_{i}=t_{1}+(i-1) h$ $(i=1, \ldots, n+1), \mathbf{x}\left(t_{i}\right)$ is approximated by NewtonCotes formulas according to Ding et al. [1].

$$
\begin{aligned}
\mathbf{x}\left(t_{i}\right)= & \mathrm{e}^{\mathbf{A}\left(t_{i}\right) \cdot\left(t_{i}-t_{i-1}\right)} \mathbf{x}\left(t_{i-1}\right) \\
& +\sum_{j=1}^{s} \frac{t_{i}-t_{i-1}}{2}\left[\mathrm{e}^{\mathbf{A}\left(t_{i}\right) \cdot\left(t_{i}-t_{i-1}\right)} \mathbf{B}_{j} \mathbf{x}\left(t_{i-1}-\tau_{j}\right)\right. \\
& \left.+\mathbf{B}_{j} \mathbf{x}\left(t_{i}-\tau_{j}\right)\right] .
\end{aligned}
$$

Since the Newton-Cotes formula has the local truncation error $\mathcal{O}\left(h^{3}\right)$, the second term of right-hand side of Eq. (5) has the local truncation error $\mathcal{O}\left(h^{3}\right)$. Therefore, the discretization error of the proposed method is $\mathcal{O}\left(h^{3}\right)$, which can also be verified via the convergence of critical eigenvalues.

Equation (39) in Sect. 3 can be expressed in the integral form when $\mathbf{A}(t)$ is a constant matrix

$$
\begin{aligned}
\mathbf{x}(t)= & \mathrm{e}^{\mathbf{A}(t) \cdot\left(t-t_{1}\right)} \mathbf{x}\left(t_{1}\right) \\
& +\int_{t_{1}}^{t} \mathrm{e}^{\mathbf{A}(t) \cdot(t-\xi)}\left[\int_{-\sigma}^{0} \mathbf{B}(\theta) \mathbf{x}(\xi+\theta) \mathrm{d} \theta\right] \mathrm{d} \xi .
\end{aligned}
$$

The period interval $T$ is also equally discretized with a time step $h$. At each small subinterval $\left[t_{i-1}, t_{i}\right]$, Eq. (40) is represented as 


$$
\begin{aligned}
\mathbf{x}(t)= & \mathrm{e}^{\mathbf{A}(t) \cdot\left(t-t_{i-1}\right)} \mathbf{x}\left(t_{i-1}\right) \\
& +\int_{t_{i-1}}^{t} \mathrm{e}^{\mathbf{A}(t) \cdot(t-\xi)}\left[\int_{-\sigma}^{0} \mathbf{B}(\theta) \mathbf{x}(\xi+\theta) \mathrm{d} \theta\right] \mathrm{d} \xi, \\
& t \in\left[t_{i-1}, t_{i}\right] .
\end{aligned}
$$

It is assumed that $\mathbf{A}(t)$ is a constant matrix on the small interval $t \in\left[t_{i-1}, t_{i}\right]$. For simplicity, $\mathbf{A}(t)$ can be replaced with $\mathbf{A}\left(t_{i}\right)$ as an approximation. At the corresponding discretized time points $t_{i}=t_{1}+(i-1) h$ $(i=1, \ldots, n+1)$, we approximate $\mathbf{x}\left(t_{i}\right)$ by using Newton-Cotes formulas, leading to

$$
\begin{aligned}
\mathbf{x}\left(t_{i}\right)= & \mathrm{e}^{\mathbf{A}\left(t_{i}\right) \cdot\left(t_{i}-t_{i-1}\right)} \mathbf{x}\left(t_{i-1}\right)+\int_{t_{i-1}}^{t_{i}} \mathrm{e}^{\mathbf{A}\left(t_{i}\right) \cdot\left(t_{i}-\xi\right)} \\
& {\left[\int_{-\sigma}^{0} \mathbf{B}(\theta) \mathbf{x}(\xi+\theta) \mathrm{d} \theta\right] \mathrm{d} \xi, \quad t \in\left[t_{i-1}, t_{i}\right] } \\
\approx & \mathrm{e}^{\mathbf{A}\left(t_{i}\right) \cdot h} \mathbf{x}\left(t_{i-1}\right) \\
& +\frac{h}{2}\left\{\left.\left[\int_{-\sigma}^{0} \mathbf{B}(\theta) \mathbf{x}(t+\theta) \mathrm{d} \theta\right]\right|_{t=t_{i}}\right. \\
& \left.+\left.\mathrm{e}^{\mathbf{A}\left(t_{i}\right) \cdot h} \cdot\left[\int_{-\sigma}^{0} \mathbf{B}(\theta) \mathbf{x}(t+\theta) \mathrm{d} \theta\right]\right|_{t=t_{i-1}}\right\} .
\end{aligned}
$$

However, it does not affect the results and conclusions of the paper. The authors would like to apologize for any inconvenience caused to the readers.

\section{Reference}

1. Ding, Y., Zhu, L.M., Zhang, X.J., Ding, H.: Numerical integration method for prediction of milling stability. J. Manuf. Sci. Eng. 133(3), 031005 (2011) 